\title{
Hybrid Job Scheduling for Improved Cluster Utilization
}

\author{
Ismail Ari and Ugur Kocak \\ Computer Engineering Department, Ozyegin University, Istanbul, Turkey
}

\begin{abstract}
In this paper, we investigate the models and issues as well as performance benefits of hybrid job scheduling over shared physical clusters. Clustering technologies that are currently supported include MPI, Hadoop-MapReduce and NoSQL systems. Our proposed scheduling model is above the cluster-specific middleware and OS-level schedulers and it is complementary to them. First, we demonstrate that we can effectively schedule MPI, Hadoop, NoSQL jobs together by profiling them and then co-scheduling. Second, we find that it is better to schedule cluster jobs with different job characteristics together (CPU vs. I/O intensive) rather than two CPU-intensive jobs. Third, we use the learning outcome of this principle to design of a greedy sort-merge scheduler. Up to $37 \%$ savings in total job completion times are demonstrated. These savings are directly proportional to the cluster utilization improvements.
\end{abstract}

\section{Introduction}

In many universities and research centers scientists depend on shared computing clusters for their computational needs. These scientists are usually from various disciplines such as Physics, Mechanical Engineering, and Computer Science. Some of their jobs are CPU-intensive and some are data-intensive. They also use different distributed processing engines and associated middleware such as MPI 12. Hadoop-MapReduce [3], and NoSQL $<k e y$, value $>$ engines to execute their jobs. As a worst case scenario (which is common in smaller scientific communities), people email each other to decide who runs a job first, which causes poor cluster utilization, or they all submit their jobs simultaneously leading to clashes and poor performance. Today, there is no easy-to-use, common scheduler to reorder and reprioritize these hybrid jobs to make better execution plans among different clustering technologies. In this paper, we describe the design of a new MPI, Hadoop-MapReduce, and NoSQL hybrid job scheduler that uses jobs' execution profiles from prior runs and makes global scheduling decisions to improve overall system utilization and job throughput performance.

\subsection{Challenges and Potentials in Hybrid Scheduling}

In this context, the word hybrid can have multiple meanings:

1. First, the interfaces or APIs of distributed processing technologies can be different (e.g. MPI vs. MapReduce) and these different middleware will be executed together on one physical platform. 
2. Second, the cluster's physical resources can be heterogeneous consisting of relatively powerful and weaker machines.

3. Third, the new overlay hybrid scheduler should know that there are also other underlying cluster-specific job schedulers and operating system (OS)level process schedulers, which creates a multi-level scheduling scenario.

The ideal hybrid scheduler should have the ability to make a combined scheduling decision considering all of the conditions above. Therefore, it would be fair to state that hybrid job scheduling is hard. Yet, we also observe the fact that in scientific communities similar jobs are run repeatedly over the same shared physical clusters as shown in Figure 1. Therefore, there is an opportunity to profile these different jobs in terms of their CPU, memory, disk bandwidth, and network usage and feed this information to a global hybrid scheduler for optimization. The clear benefits of hybrid job scheduling include increased resource utilization, convenience on the user's side, and better job isolation. This paper aims to investigate and highlight some of these potential advantages.

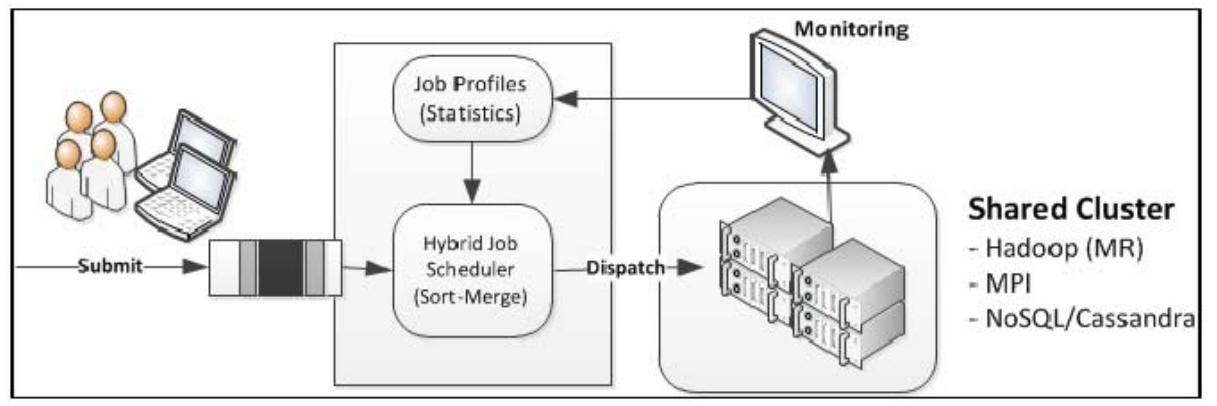

Fig. 1. A hybrid job scheduler for shared physical clusters collects and uses job profiles or statistics from prior runs

In one aspect, the hybrid job scheduler virtualizes the underlying clustering technologies, creating a unified scientific cloud platform service (or PaaS). The underlying cluster infrastructure can be physical or virtual or deployed as a private or public infrastructure cloud service (i.e. IaaS). The end users do not have to know these details. In addition, they also would not have to manage the cluster middleware, i.e. know whether MPI, Hadoop, NoSQL (HBase 4], Cassandra [5], MongoDB [6]) are installed properly. One scientist running High Performance Computing (HPC) jobs only may not fully utilize all the cluster resources (e.g. $100 \%$ of the CPU, but no disk bandwidth), but when heterogeneous tasks from different departments are put together better cluster utilization is achievable.

\section{Background - Big Data, Hadoop, MPI, NoSQL}

The need for clusters have been driven primarily by HPC applications in the past. Today, there is an emergence of High Throughput Computing (HTC) [1920] 
applications that contend for the same cluster resources. Many organizations including universities, telecommunication operators, media \& social networks, financial institutions and governments generate Terabytes of data each day and attempt to insert this high "volume" data into databases that already contain Petabytes. Data come from different sources such as sensors, various web/system logs, and mobile phone call detail records (CDR). The mixing of these different data types results in a wide "variety" of data to be processed and stored (e.g. unstructured text, semi-structured XML-JSON, structured CSV, or binary audio/video data). The term "Big Data" is used to refer to challenging data management problems that arise due to the high Volume, Velocity, Variety, and Veracity (4Vs) of the data 7. Relational database management systems cannot cope with $4 \mathrm{Vs}$ of data very well, therefore Hadoop and NoSQL systems have quickly penetrated into the scientific and enterprise data infrastructures. Consequently, a variety of the cluster technologies started to get used over the same, shared physical clusters.

\subsection{Hadoop}

Apache Hadoop is an open-source framework written in Java designed for supporting data-intensive distributed applications. Hadoop framework mainly consists of two components called MapReduce (MR) and Hadoop Distributed File System (HDFS) as shown in Figure 2. The data to be processed is stored in large fixed-sized (64MB) chunks by HDFS for performance and reliability reasons. HDFS consists of a NameNode and DataNodes. MapReduce (MR) is a distributed job execution system consisting of a JobTracker and TaskTrackers. Jobs will be split into many Map and Reduce tasks and sent as JAR files to many machines containing the data in HDFS. Chen, et al. 8] give a detailed analysis of MR workloads from two large-scale production Hadoop systems (Yahoo \& Facebook) and find great differences between MR use cases. They conclude that "no single benchmark can capture such diverse behavior" [8].
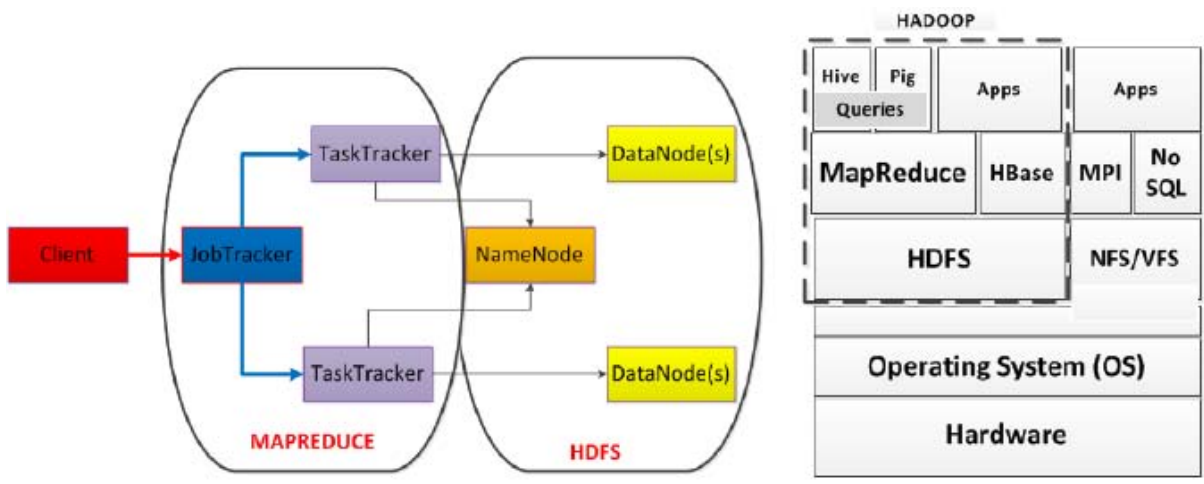

Fig. 2. (Left) Hadoop architecture overview and (right) diagram showing colocation of Hadoop, MPI and NoSQL systems in one machine 


\subsection{Message Passing Interface (MPI)}

MPI is an HPC middleware used for distributed inter-process communication. It is well-standardized and portable. We use the MPICH2 [1 implementation of the standard, but other implementations such as the Open MPI 2 also exist. In our previous HPC-focused work 9, we implemented a cloud computing platform service for Finite Element Analysis (FEA). We found that if the FEA job can be processed effectively on a single multi-core server (i.e. with enough CPU cores and RAM), we should use the SMP-style multi-processing. If not, the job will be distributed over multiple servers/processes and MPI will be used for interprocess communication. The scheduler has to make these decisions optimally for hundreds of jobs, simultaneously [9].

\subsection{NoSQL - Apache Cassandra}

The most commonly used NoSQL systems include HBase [4, Cassandra [5, and MongoDB [6. We selected Cassandra and continued with it as our NoSQL system. Apache Cassandra [5] is a Java-based NoSQL database initially developed by Facebook. One of its differentiators is that, it has no central servers and uses a pure peer-to-peer approach for scalability. Cassandra does not enforce a schema, thus it is also a $<k e y$, value $>$ store like Amazon's Dynamo [10] system. The "table" concept in DBMS is represented by a "column family" in Cassandra. Cassandra opens a thread per worker thread. However, it also uses additional CPU resources for other background jobs, like indexing and flushing, which shows as random spikes in its CPU profile.

\section{Related Work - Cluster Job Scheduling}

While most state-of-the-art cluster job scheduling tools declare heterogeneous system support, they primarily refer to different hardware capabilities and not middleware interfaces. For HPC jobs, the famous MPI scheduler called LSF (Load Sharing Facility) [11 is primarily used for submitting MPI jobs to MPI clusters. The scheduler adjusts execution order of submitted MPI tasks based on their node-core ("-np") requirements and their priorities. In our previous work [9, we showed that additional savings are possible above the MPI-level with task-aware cluster job scheduling especially for FEA [9]. In this paper, we demonstrate potential savings for hybrid set of jobs, and not just HPC. Previous research on Grid scheduling and scientific workflows focuses on data or task dependencies 14, [15. Since our jobs belong to completely different middleware we assume there will be no such dependencies and issues among our hybrid jobs. There are numerous past publications on Grid scheduling, but since none of these prior works support Hadoop, MPI and NoSQL we skip a detailed comparison here for brevity. For HTC jobs, there exists two Hadoop schedulers called FIFO and Fair Scheduler [12. Using these schedulers and careful job pool settings, prioritized co-scheduling of MapReduce jobs is possible. However, these Hadoop schedulers do not accept MPI jobs today. 
We are not aware of any system that integrates MPI, Hadoop, and NoSQL systems as we do in this paper. The closest systems to ours include the SLURM Scheduler plugins and HTCondor ClassAds. "SLURM is an open-source resource manager designed for Linux clusters" 24. It provides a framework for allocating, starting, executing, and monitoring work (typically a parallel job) on a set of allocated nodes for a specified duration. SLURM's modular design accepts plugins inluding scheduler plugins that implement the SLURM scheduler API. To name a few, builtin scheduler plugin implements FIFO and backfill raises the priority of jobs to start them earlier. "HTCondor's ClassAds are a flexible mechanism for representing the characteristics and constraints of machines and jobs in the HTCondor system" 25. HTCondor currently has no direct MapReduce support.

One could simply use native or hosted machine virtualization to co-locate different clustering middleware (or platforms) on shared physical cluster infrastructure. In comparsion, our proposed hybrid job scheduling model has one of the simplest forms. In our proposed model, different clusters are placed under the common scheduler, which profiles all the submitted jobs and decides to colocate (i.e. dispatch together) those who use different, heterogeneous types of resources. In this regard, the hybrid job scheduler virtualizes the underlying clustering middleware, creating a unified scientific cloud. There are two other cases called Hadoop over MPI or MPI over Hadoop, where the former refers to converting MapReduce source or binary code using an adapter to MPI code to run over an MPI cluster 21] and the latter refers to the new YARN approach mentioned in Section 2.1. There can also be native and a hosted virtualized models, for mixed Hadoop-MPI virtual clusters. These two virtualization alternatives are quite similar with respect to the higher-layer job scheduling software. However, the natively virtualized clusters provide additional advantages over hosted VMs including dynamic resizing and migration of VMs, and scale easily beyond a single physical machine. As tradeoffs, virtualized clusters have both performance overheads and additional VM management issues [18.

Recent findings [22] also suggests that virtualization may not always be the answer in the HPC world for multiple reasons. First, HPC systems want to squeeze the last bit of performance from the systems whereas virtualization can mean up to $10 \%$ of performance hit, since the hypervisor adds yet another layer between the physical resources and the applications. The second reason is HPC systems are already designed for being shared among a community of people in unified scientific communities (i.e. with similar HPC jobs) the utilizations can be relatively high 22 . Therefore, the motivations that drive the use of virtualization (i.e. increase utilization, save power and same money) are not always there for HPC. Similarly for Hadoop clusters, experts neither recommend virtualization nor RAID, but to save the money and buy more nodes and disk spindles. While the fastest $15 \mathrm{~K}$ rpm disks are common in $\mathrm{HPC}$, they are not recommended in Hadoop and other HTC clusters, as one can get more parallel I/O bandwidth by buying more $7200 \mathrm{rpm}$ disks. In summary, virtualization is a good choice in dynamic test and development environments where reconfiguration speeds and 
elasticity are critical. However, HPC and HTC environments run similar and predictable types of workloads and require deterministic performance.

\section{Performance Analysis and Results}

The experiments for this demo were conducted on a small cluster of two IBM HS22 Blade Servers in an HPC blade chassis with 1Gbps connectivity. Each server has two $2.40 \mathrm{GHz}$ Intel Xeon Quad-Core CPUs, 24GB Memory, and two 72 GB 15000rpm disks configured as RAID1. We installed RedHat Linux 5 on these servers. For profiling we used the sysstat package [16] and its pidstat command to track CPU, memory and disk read-write bandwidth usage of the processes. All experiments were run 3 times and the results were averaged.

\subsection{Workloads}

As Hadoop workload we used the classical terasort benchmark, which is more IO-intensive than our MPI task. For this experiment, we first created a large (15 GB) file filled with random, unsorted characters using the teragen program. Next, we used terasort to read \& sort the unsorted data and write back the sorted data. Hadoop version 1.0.2 was used. As MPI workload we used the dbworld benchmark, which solves a system of linear equations. The MPI command was executed as follows: <mpirun -np 8 dbworld_mpi -n 1536 -m 1536>, where np denotes node-core counts and $n \times m$ are the matrix row-column sizes. We used the MPICH2 implementation [1] of MPI. During Hadoop comparison, matrix size was asjusted to $1348 \times 1348$, so that one task does not finish much sooner. This simulates a scenario where the job pipeline is always full.

For Cassandra benchmarking, we wrote a Java program that reads the XMLformatted CDR logs of a real mobile telecom company, indexes and inserts these transformed logs into Cassandra using the Astyanax client ( JDBC) by Netflix. For data distribution we used random sharding technique and no replication. Table 1 shows the average CPU-memory usage \%, and read-write bandwidth usage in $\mathrm{MB} /$ second for these 3 workloads obtained using the pidstat tool. Basically, the MPI and Cassandra benchmark jobs were CPU-intensive (with $99 \% \mathrm{CPU}$ utilization on average) and the Hadoop terasort job was more IO-intensive than others $(10 \mathrm{MB} / \mathrm{sec}$ reads, $40 \mathrm{MB} / \mathrm{sec}$ writes) and had $25 \%$ average CPU usage.

Table 1. Resource usage profiles of benchmark cluster workloads

\begin{tabular}{|l|l|l|l|}
\hline & CPU & Memory & R-W Bandwith \\
\hline MPI & $99 \%$ & $0.5 \%$ & $0-0 \mathrm{MB} / \mathrm{sec}$ \\
\hline Cassandra & $99 \%$ & $15 \%$ & $0-4.3 \mathrm{MB} / \mathrm{sec}$ \\
\hline Hadoop & $25 \%$ & $2.2 \%$ & $9.8-40.2 \mathrm{MB} / \mathrm{sec}$ \\
\hline
\end{tabular}


Our focus in this paper was to mix and run different types of jobs together for different middleware, therefore we only analyzed interactions among jobs for multiple core count settings and did not vary the system-specific parameters internally. If we vary the size of either one of the benchmarks (MPI problem size, terasort data size, or Cassandra data size), their executions times will be longer, but the learning outcomes will be the same.

\subsection{CPU-intensive Jobs and Cluster-Level Job Prioritization}

Table 2 shows the results of co-scheduling of two CPU-intensive tasks MPI and Cassandra on a single 8-core (physical) server for different worker $(4,8,16)$ settings. For MPI, the term "worker" refers to the number of cores used (i.e. -np) and for Cassandra it refers to the number of loader client threads. "Standalone" refers to non-overlapping, i.e. serial and mutually exclusive, execution of these two tasks. With 4 workers the total time to finish both tasks is 20.8 minutes $(11.8+9.0)$. With 8 workers the total time goes down to 15.4 minutes $(\sim 26 \%$ improvement), but does not improve anymore for 16 workers since hyperthreading does not contribute much for $\sim 100 \%$ CPU-intensive tasks. The Cassandra task benefits more from increased core count.

When the two tasks are started simultaneously with the same priorities and they execute in parallel, the OS-level process scheduler shows positive effect. Although both tasks execute longer than their serial execution counterparts (e.g. 16.2 mins for MPI and 10.1 mins for Cassandra 4) the system throughput increases from $\sim 6$ jobs/hour to $\sim 8$ jobs/hour: both tasks have completed at 16.2 minutes for 4 workers, 15.0 minutes for 8 , and 13.8 minutes for 16 workers. This shows that 10-20\% savings are possible even for CPU-intensive tasks.

Next, we change the priorities of the tasks using the Linux nice command to see if we can control the CPU-shares at the cluster level at run-time. In Cassandra, we change the priority of the server, whereas in MPI the priority of the job is directly changed. -20 and +19 are the highest and lowest priorities, respectively. The results are affirmative in terms of job control. The job with higher priority can perform comparable to its standalone performance, whereas the niced job has to wait (or run slow) until the high-priority job completes. This demonstrates that we can order, prioritize, or re-prioritize jobs belonging to different cluster technologies with respect to each other.

\subsection{Hybrid Scheduling of CPU and IO-intensive Jobs}

Table 3 shows the serial and hybrid execution of MPI and Hadoop jobs. We used the 15 GB data for the Hadoop terasort benchmark job. The serial execution completed in 1272 seconds total, whereas the hybrid execution completed around 800 seconds. This results in savings of $\sim 37 \%$. The savings are significantly higher compared to co-scheduling of two CPU-intensive jobs, which resulted in 10-20\% savings. Note that the execution time of the CPU-intensive MPI job is $\sim 20 \%$ higher (800 sec.) in hybrid form (i.e. MPI-Hadoop co-scheduled) compared to its serial execution time (671 sec). The Hadoop job also took $\sim 25 \%$ longer, but 
Table 2. Results of co-scheduling MPI and Cassandra jobs

\begin{tabular}{|c|l|l|l|l|}
\hline Worker\# Per-Job & Execution & $\begin{array}{l}\text { MPI } \\
(\mathrm{min})\end{array}$ & $\begin{array}{l}\text { Cassandra } \\
(\mathrm{min})\end{array}$ & $\begin{array}{l}\text { Total } \\
(\mathrm{min})\end{array}$ \\
\hline \multirow{4}{*}{4} & standalone & 11.8 & 9 & 20.8 \\
& normal - combined & 16.2 & 10.1 & 16.2 \\
& nice -n -20 for M & 14.9 & 18.4 & 18.4 \\
& nice -n +19 for M & 18.4 & 10.5 & 18.4 \\
& nice -n -20 for C & 19.9 & 10.5 & 19.9 \\
& nice -n +19 for C & 13.4 & 17.7 & 17.7 \\
\hline \multirow{5}{*}{8} & standalone & 9.4 & 6 & 15.4 \\
& normal - combined & 15 & 8.6 & 15 \\
& nice -n -20 for M & 11.8 & 16.3 & 16.3 \\
& nice -n +19 for M & 17.6 & 7.5 & 17.6 \\
& nice -n -20 for C & 16.8 & 7.8 & 16.8 \\
& nice -n +19 for C & 10 & 16.1 & 16.1 \\
\hline \multirow{3}{*}{16} & standalone & 9.5 & 5.8 & 15.3 \\
& normal - combined & 13.8 & 10.7 & 13.8 \\
& nice -n -20 for M & 11.3 & 16 & 16 \\
& nice -n +19 for M & 16.5 & 6.6 & 16.5 \\
& nice -n -20 for C & 15.6 & 6.8 & 15.6 \\
& nice -n +19 for C & 10.6 & 15.4 & 15.4 \\
\hline
\end{tabular}

Table 3. Results of co-scheduling of MPI and Hadoop jobs

\begin{tabular}{|l|l|l|l|}
\hline & MPI $(\mathrm{sec})$ & Hadoop $(\mathrm{sec})$ & Tot. Time $(\mathrm{sec})$ \\
\hline Serial Exec. & 671.7 & 601.0 & 1272.7 \\
\hline Hybrid Sched. & 800.3 & 769.0 & 800.3 \\
\hline
\end{tabular}

they both finished at 800.3 seconds. These job latency increases can be attributed to the $20-25 \%$ on average CPU usage of the Hadoop job.

\section{Generalized Hybrid Scheduler}

Now, we briefly describe a greedy sort-merge hybrid job scheduler. First, jobs are profiled based on their resource usage. Job $i\left(j_{i}\right)$ is represented by a triplet $j_{i}=<c_{i}, m_{i}, d_{i}>$, where $c_{i}, m_{i}$, and $d_{i}$ represents the average CPU, memory and read+write disk I/O usage of that job, respectively. Clients use our "hsub" command (stands for hybrid submit) and is similar to the LSF qsub-bsub commands. Sample hsub commands are given below:

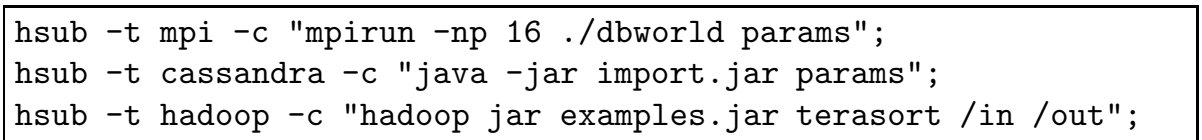

where -t denotes the job type (mpi,hadoop,cassandra) and -c parameter gives the command string (parameters are omitted). 


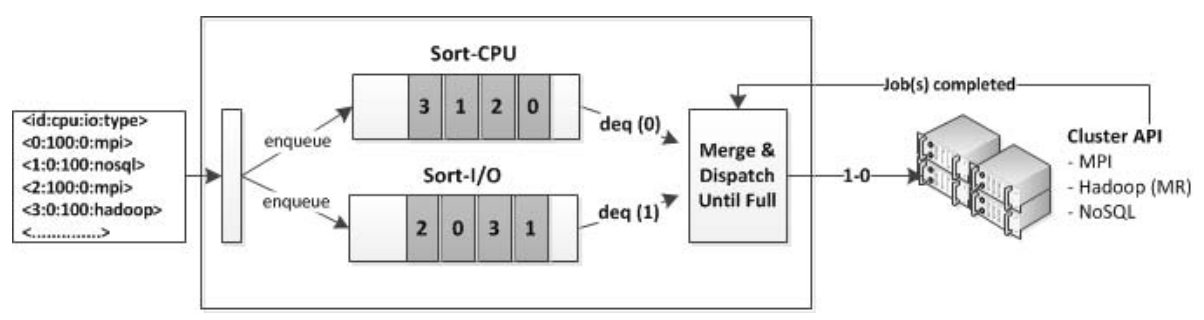

Fig. 3. Greedy sort-merge scheduler

Figure 3 illustrates the operation of this scheduler with a simple example. Jobs belonging to different clusters are submitted to the scheduler by various clients. In this example, a CPU-intensive job will be followed with an IO-intensive job. The scheduler keeps separate lists for each resource (CPU, disk, memory) in the decreasing order of their usage of that particular resource. Jobs are enqueued to all of the lists and then dequeued from the lists in a round-robin fashion. The corresponding capacities are subtracted from the resources, respectively. This way, a CPU-intensive job will first be matched with a disk-intensive job and so on until all jobs are complete. We generated two workloads: first, an HPCHTC mix (i.e. CPU-intensive and IO-intensive mix) and second, a completely random mix on all dimensions (cpu, memory, disk). We compared different job sorting strategies using 1000 simulated jobs. We have written about 500 lines of Java code for the hybrid scheduler simulation. We briefly report the results here. Since the HPC-HTC mix was generated for an ideal case of $100 \% \mathrm{CPU}$ intensive $(<100: 0: 0>)$ and 100\% IO-intensive $(<0: 0: 100>)$ mix, the greedy scheduler was able to finish 1000 such jobs in 500 epochs. That is $2 \mathrm{x}$ performance improvement (50\% savings in total time) over a single CPU-based or a single IO-based sorted scheduler. For the random mix, the two schedulers have similar performance around 900 epochs each.

\section{Conclusions}

In this paper, we analyzed different models for hybrid job scheduling (above middleware and VM-based) over shared physical clusters. Our proposed scheduling layer is above the cluster middleware and OS-level schedulers. We were able to improve cluster utilization and overall throughput (jobs/min) with some increase in per job latency. Our important findings were as follows: (1) We can schedule jobs that belong to different clustering technologies (Hadoop, NoSQL, MPI) together effectively. (2) We found that two CPU-intensive jobs, that can each fully utilize the multi-core and multi-node resources of the cluster can still benefit from co-scheduling, but it is better to schedule I/O and disk-intensive jobs together first. (3) We demonstrated 10-37\% decrease in total job processing times. This means that by continuous pipelining of heterogenous job types we can sustain up to $37 \%$ improvement in cluster utilization. 
Acknowledgment. This work was supported in part by European Union FP7 Marie Curie Program BI4MASSES Grant, Turkish National Institute of Science and Technology (TUBITAK) Grant 190E194, IBM Shared University Research (SUR) program, and by Turkish Telecomm and Avea Labs.

\section{References}

1. MPICH project, http://www.mpich.org

2. Open MPI project, http://www.open-mpi.org

3. Apache Hadoop Project, http://hadoop.apache.org/

4. Apache Hbase project, http://hbase.apache.org

5. Apache Cassandra, http://cassandra.apache.org/

6. MongoDB by 10gen, http://www.mongodb.org/

7. What is Big Data? http://www-01.ibm.com/software/data/bigdata/

8. Chen, Y., Ganapathi, A., Griffith, R., Katz, R.: The case for evaluating MapReduce performance using workload suites. In: 2011 IEEE 19th International Symposium on Modeling, Analysis \& Simulation of Computer and Telecommunication Systems (MASCOTS), pp. 390-399 (2011)

9. Ari, I., Muhtaroglu, N.: Design and implementation of a cloud computing service for finite element analysis. Advances in Engineering Software 60-61, 122-135 (2013)

10. DeCandia, G., Hastorun, D., Jampani, M., Kakulapati, G., Lakshman, A., Pilchin, A., Sivasubramanian, S., Vosshall, P., Vogels, W.: Dynamo: amazon's highly available key-value store. SOSP 7, 205-220 (2007)

11. Zhou, S., Zheng, X., Wang, J., Delisle, P.: Utopia: a load sharing facility for large, heterogeneous distributed computer systems. Software: Practice and Experience 23(12), 1305-1336 (1993)

12. Hadoop Fair Scheduler, http://hadoop.apache.org/docs/stable/fair_ scheduler.html

13. Etsion, Y., Tsafrir, D.: A short survey of cluster batch schedulers, Technical Report, Hebrew University, 2005-13 (May 2005)

14. Deelman, E.: Grids and Clouds: Making workflow applications work in heterogeneous distributed environments. International Journal of High Performance Computing Applications 24(3), 284-298 (2010)

15. Juve, G., Deelman, E.: Resource provisioning options for large-scale scientific workflows. In: Proc. of SWBES Workshop with IEEE E-Science (2008)

16. Sysstat Utils, sebastien.godard.pagesperso-orange.fr/

17. Dhiman, G., Marchetti, G., Rosing, T.: vGreen: a system for energy efficient computing in virtualized environments. In: Proceedings of the 14th ACM/IEEE International Symposium on Low Power Electronics and Design, pp. 243-248. ACM

18. Hu, L., Schwan, K., Gulati, A., Zhang, J., Wang, C.: Net-cohort: Detecting and managing vm ensembles in virtualized data centers. In: Proceedings of the 9th International Conference on Autonomic Computing, pp. 3-12. ACM (2012)

19. Zhan, J., Zhang, L., Sun, N., Wang, L., Jia, Z., Luo, C.: High Volume Throughput Computing: Identifying and Characterizing Throughput Oriented Workloads in Data Centers. In: 2012 IEEE 26th International Parallel and Distributed Processing Symposium (2012) 
20. Hwang, K., Fox, G.C., Dongarra, J.J.: Distributed and Cloud Computing, From parallel processing to Internet of things. Morgan Kaufman, Elsevier (2012)

21. Plimpton, S.J., Devine, K.D.: MapReduce in MPI for large-scale graph algorithms. Parallel Computing Journal 37, 610-632 (2011)

22. HPC Virtualization, Virtualization in HPC may not always be the answer as Gillian Law discovers, Scientific Computing World, pp. 21-24 (October/November 2011)

23. Novotny, M.: Job scheduling with the SLURM resource manager, Bachelor Thesis, Masarykova Univerzita

24. SLURM Workload Manager, http://slurm.schedmd.com/

25. HTCondor(tm) Version 7.8.8 Manual, http://research.cs.wisc.edu/htcondor/ manual/v7.8/ 